\title{
Humanized Mouse Models for the Study of Human Malaria Parasite Biology, Pathogenesis, and Immunity
}

\author{
Nana K. Minkah', Carola Schafer ${ }^{1}$ and Stefan H. I. Kappe ${ }^{1,2 *}$ \\ ${ }^{1}$ Center for Infectious Disease Research, Seattle, WA, United States, ${ }^{2}$ Department of Global Health, University of \\ Washington, Seattle, WA, United States
}

Malaria parasite infection continues to inflict extensive morbidity and mortality in resource-poor countries. The insufficiently understood parasite biology, continuously evolving drug resistance and the lack of an effective vaccine necessitate intensive research on human malaria parasites that can inform the development of new intervention tools. Humanized mouse models have been greatly improved over the last decade and enable the direct study of human malaria parasites in vivo in the laboratory. Nevertheless, no small animal model developed so far is capable of maintaining the complete life cycle of Plasmodium parasites that infect humans. The ultimate goal is to develop humanized mouse systems in which a Plasmodium infection closely reproduces all stages of a parasite infection in humans, including pre-erythrocytic infection, blood stage infection and its associated pathology, transmission as well as the human immune response to infection. Here, we discuss current humanized mouse models and the future directions that should be taken to develop next-generation models for human malaria parasite research.

Keywords: Plasmodium falciparum, humanized mouse models, FRG human hepatocyte, human immune system mice, malaria vaccines

\section{MALARIA: DISEASE BURDEN, PARASITE LIFE CYCLE, NATURAL IMMUNITY, AND THE DEVELOPMENT OF A MALARIA VACCINE}

Specialty section:

This article was submitted

to Vaccines and

Molecular Therapeutics,

a section of the journal

Frontiers in Immunology

Received: 15 December 2017

Accepted: 03 April 2018

Published: 19 April 2018

Citation:

Minkah NK, Schafer C and Kappe SHI (2018) Humanized

Mouse Models for the Study of Human Malaria Parasite Biology,

Pathogenesis, and Immunity.

Front. Immunol. 9:807. doi: 10.3389/fimmu.2018.00807

Malaria, a disease caused by protozoan Plasmodium parasites, causes more than 200 million clinical cases annually and is responsible for more than 400,000 deaths each year, mainly in children under the age of 5 and pregnant women living in the resource-poor countries of sub-Saharan Africa. In humans, the majority of malaria infections are caused by Plasmodium falciparum and Plasmodium vivax. In more temperate regions of the world, socioeconomic development, vector control programs, and the use of antimalarial chemotherapeutics have driven successful malaria elimination. However, declines in malaria infections have been slowest in tropical, resource-poor countries with a high malaria burden necessitating the development of new effective antimalaria therapeutics and vaccines that will prevent infection, disease, and onward transmission.

Transmission of malaria parasites to the mammalian host begins with the deposition of the infectious, motile sporozoite stages into the skin via the bite of infected mosquitoes (1-3). Sporozoites initially traverse multiple cell types in the skin in search of capillaries to gain access to the bloodstream within which they are transported to the liver (3). Each sporozoite infects a hepatocyte, then transforms into a liver stage that undergoes growth, genome replication, and differentiation into tens of thousands of red blood cell (RBC) infectious exo-erythrocytic merozoites. In humans, 


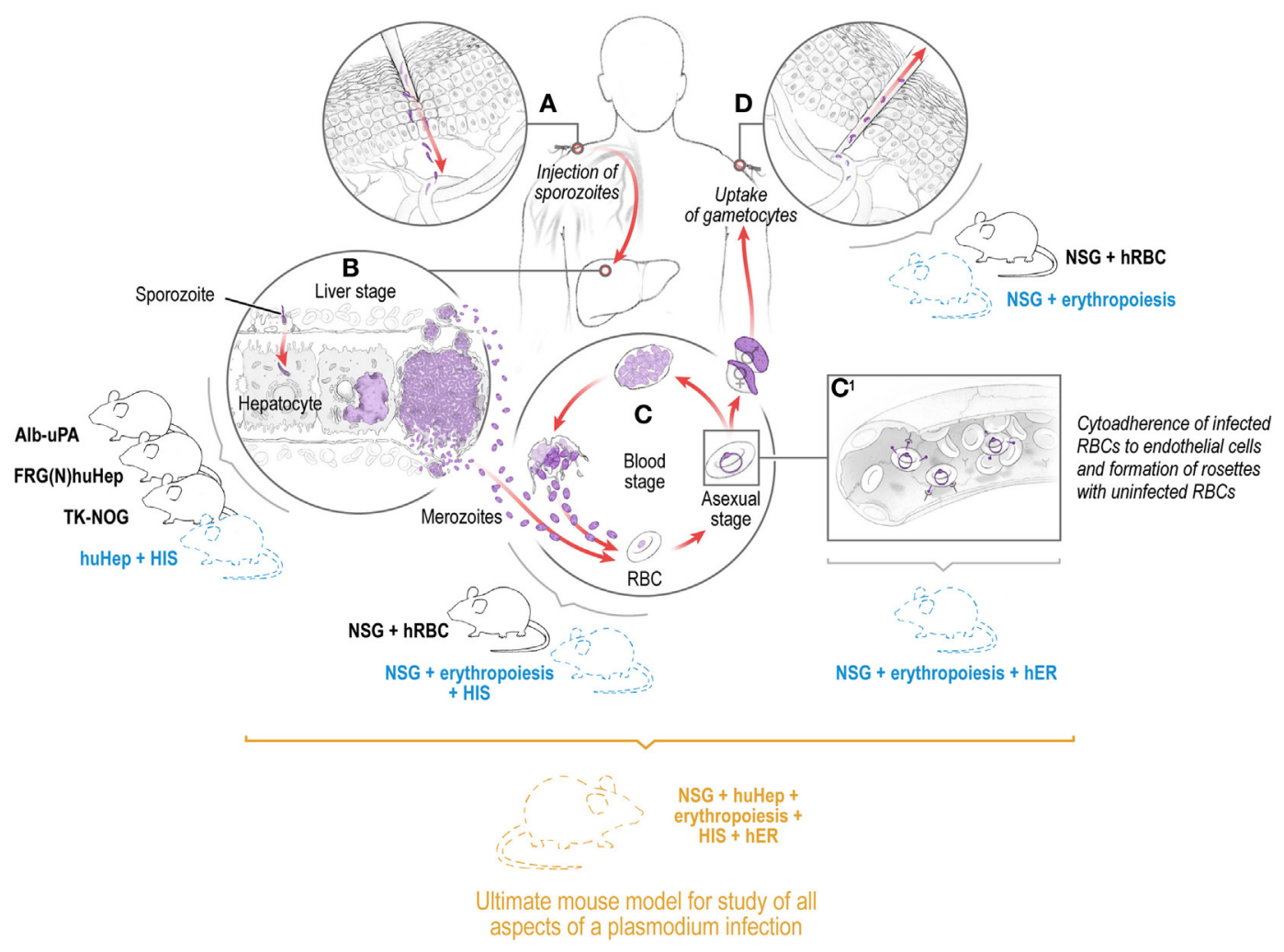

FIGURE 1 | Depiction of the Plasmodium life cycle in humans showing the skin, liver, and blood stages with the corresponding existing (solid lines) and future (dashed lines) humanized mouse systems to model each of the individual stages and eventually the full Plasmodium life cycle including pathology and transmission. Infection is initiated when a female Anopheles mosquito injects saliva-containing sporozoites into the skin. Sporozoites traverse dermal cells and gain access to the blood (A). The highly motile sporozoites transit to the liver where each sporozoite infects a single hepatocyte (B). One to two weeks after hepatocyte invasion, merozoites exit the liver and begin a 48-h cycle of red blood cell (RBC) invasion, replication, RBC rupture, and new merozoite release (C). During RBC infection, the parasite expresses variant surface antigens on the surface of the infected red blood cell, which interacts with human endothelial receptors (hER), thus mediating the binding of infected RBCs to the microvascular endothelium of various organs ( $\mathbf{C}^{1}$ ). A small number of blood-stage parasites differentiate into sexual gametocytes, which are taken up by mosquitoes in blood meals to continue the transmission to new human hosts (D).

merozoites are released from the liver into the bloodstream 7-10 days after initial transmission where they infect RBCs, replicate within, and are released to undergo continuous cycles of infection, replication, and release, allowing parasite numbers to reach billions within weeks (Figure 1). In P. vivax infection, a subset of sporozoites form persistent liver stages (hypnozoites) that activate at later time points to cause relapsing blood stage (BS) infections $(4,5)$. All symptoms associated with malaria are caused by BS infection, and this is in large part due to the massive destruction of RBCs but also the sequestration of infected RBCs in the microvasculature (6). This sequestration can occur in a tissue-specific manner, leading, for example, to cerebral malaria pathology through sequestration of infected RBCs in the brain or pregnancy associated malaria due to sequestration of infected RBCs in the placenta. Uptake of parasite sexual forms in a blood meal leads to infection of the mosquito, sporogonic development, and colonization of salivary glands by sporozoites, which ensures transmission to new human hosts $(7,8)$ (Figure 1).

Repeated Plasmodium infection does not result in complete immunity, rendering populations in endemic regions continuously susceptible to infection, malaria-associated morbidity and mortality as well as transmission. A fully protective malaria vaccine has yet to be developed (9). Moreover, evolution of parasite resistance to frontline antimalarial drugs necessitates continuous research and development of next-generation antimalarials (10). These efforts require robust experimental systems that accurately model human malaria parasite biology, immunology, and pathogenesis. Given the tropism of the human Plasmodium species for human hepatocytes (huHeps) and RBCs, researchers have relied on in vitro infection models for human parasites to query malaria biology and identify targets of intervention. In this review, we will discuss how blood, tissue, and immune system-humanized mouse models can provide novel avenues to examine human malaria parasites. Humanized mice and their past use in malaria research have been reviewed recently $(11,12)$. Therefore, in this review, we will focus more on critical research gaps in our understanding of human malaria parasite biology, pathology, and immunology that might be addressed in humanized mouse models and improvements to these models that are needed to achieve this. We will also reflect on the role of next-generation multi-compartmenthumanized mice in the modeling of the complete Plasmodium life cycle in physiologically relevant human cells and tissues. 


\section{PRE-ERYTHROCYTIC (PE) Plasmodium INFECTION: BIOLOGY OF TRANSMISSION - THE SKIN AND LIVER STAGES}

The sporozoite and liver stages comprise the PE stage of infection. Unlike the BSs, the PE stages are asymptomatic, are small in numbers during natural infection, and are not as antigenically variant. These characteristics render them extremely attractive targets for malaria intervention (13). Dissimilar to the BSs, the PE stages of $P$. falciparum cannot be easily generated in the laboratory and although some aspects of human infection can be modeled in vitro, these systems have limitations. Therefore, most of our knowledge on PE biology has been derived from studies of rodent malaria parasites, which were originally isolated from wild African rodents and subsequently adapted to laboratory mice (14-16). For transmission research, the biology of skin infection between different rodent parasite species (Plasmodium yoelii and Plasmodium berghei) made it attractive to speculate that parasite behavior in the skin should be similar in Plasmodium species that infected humans. However, the recent identification of the development of exo-erythrocytic merozoites in the skin of P. berghei-infected mice (17) but not in P. yoelii-infected mice (18) identified one point of divergence even within these two closely allied parasite species. Thus, whether the development of skin exoerythrocytic merozoites occurs in human Plasmodium parasites remains an open question, as preventive therapeutics targeted to the liver stage might be ineffective in the skin. Given that this skin stage of infection can only be adequately modeled within the three-dimensional architecture of the skin tissue, recent advances in the engraftment of human skin into immunodeficient mice (19) represent an exciting opportunity to examine interaction of human parasites with human skin components in vivo and an opportunity to explore the occurrence and relevance of "skin exo-erythrocytic merozoites" in Plasmodium parasites that infect humans.

Each sporozoite that reaches the liver invades a single hepatocyte, transforms into a trophic stage, and then commences liver stage development (also called exo-erythrocytic development). Traditional rodent malaria models have been critical to the identification of host hepatocyte surface factors necessary for sporozoite invasion $(7,20)$. Recently, human liver-chimeric mice have been employed to examine the contribution of these invasion factors in human malaria parasite liver infection (21). The efficient engraftment of huHeps into mice is dependent on an environment of severe immunodeficiency to limit huHep rejection coupled with the elimination of mouse hepatocytes to provide the huHep a niche in the liver parenchyma. Three different mouse models have been utilized for huHep engraftment and have been used to assess liver stage infection by Plasmodium parasites infecting humans. The SCID Alb-uPA model expresses the toxic urokinase plasminogen activator (uPA) under the control of an albumin promoter in the livers of the highly immunodeficient Severe Combined Immune Deficiency mice (SCID Alb-uPA). Upon engraftment with huHeps, these mice become susceptible to infection with $P$. falciparum sporozoites and support complete liver stage development including the release of exo-erythrocytic merozoites that egress and invade human RBCs (huRBCs) ex vivo $(22,23)$. An alternate to the induction of hepatotoxicity by uPA transgene expression is genetic ablation of fumarylacetoacetate hydrolase (FAH) in mice resulting in acute liver failure which can be rescued by administration of the drug, 2-(2-nitro-4fluoromethlbenzoyl)-1,-3-cyclohexanedione (NTBC). Crossing these $\mathrm{FAH}^{-/-}$mice onto the severely immunocompromised $\mathrm{C} 57 \mathrm{BL} / 6$ $\mathrm{Rag}^{-/-} \mathrm{IL} 2 \mathrm{rr}^{-/-}$mouse generated $\mathrm{FAH}^{-/} \mathrm{Rag}^{--} \mathrm{IL}_{2} \mathrm{rg}^{-/-}$(FRG) mice. These mice have also been backcrossed onto the non-obese diabetic (NOD) background (FRGN), which additionally renders them hospitable to transplantation with $\mathrm{CD}_{3} 4^{+}$hematopoietic stem cells (HSCs) (24). Using NTBC cycling during engraftment, these mice can exhibit over 90\% engraftment with huHeps (FRG huHep) (25), are susceptible to infection with both P. falciparum and $P$. vivax sporozoites $(26,27)$ and support full liver stage development, including the release of exo-erythrocytic merozoites capable of invading huRBCs that were infused into the mice. When infected with P. vivax, FRG huHep mice also harbor nonreplicating hypnozoites (27). More recently, the TK-NOG (NOD/ Shi-scid/IL2 $\mathrm{rg}^{-/-}$) mouse has been developed as yet another model for P. falciparum PE infection (28). These mice express the herpes simplex virus thymidine kinase transgene under the control of the albumin promoter on the NOD SCID IL2 $\mathrm{r} \gamma^{-/-}$ background. Destruction of mouse hepatocytes is achieved by treatment with ganciclovir, allowing repopulation of the liver with huHeps. TK-NOG mice support $P$. falciparum and Plasmodium ovale sporozoite infection and liver stage development (28).

\section{Anti-PE Plasmodium IMMUNITY AND VACCINE DEVELOPMENT: FROM TRADITIONAL MOUSE MODELS TO HUMAN CLINICAL TRIAL}

The pronounced human host cell tropism of malaria parasites that infect humans precludes infection of the traditional immunology workhorse, the in-bred mouse. Thus, the rodent malaria parasites, $P$. yoelii and $P$. berghei have been extensively utilized because they allow a careful examination of PE immunity. Perhaps the greatest contribution of the rodent malaria models is in the examination of immunity and protection against an infectious sporozoite challenge after vaccination with whole, attenuated sporozoites. Attenuation was originally generated by gamma irradiation (29) but can now be achieved by genetic engineering with the precise removal of genes from the parasite genome $(30,31)$ or by treatment of an infectious sporozoite immunization with drugs that prevent BS infection $(32,33)$. Over the last decade, studies utilizing whole sporozoite infection of mice have identified roles for humoral immunity $(3,34-37)$ and both peripheral and tissueresident memory CD8 T cells in the protective response to immunization (13, 38-41). Previously thought to be immunologically silent, liver stage Plasmodium infection also induces an innate immune response (42-45). The pathways by which this innate immune response is induced and the influence it has on the ensuing adaptive immune response are areas of active investigation.

Although traditional rodent models of PE infection have been useful, differences in rodent and human Plasmodium species (46) 
compounded with significant divergence in rodent and human hosts presents significant implications for the extrapolation of results achieved with the former to the latter, particularly with regard to host-parasite interactions and immunity. Also, while the liver stages of rodent Plasmodium species develop fully within 2-3 days, the human Plasmodium parasites undergo 7-10 days of liver stage development before exiting the liver to infect erythrocytes. In addition, none of the rodent parasites form persistent liver stages that could model those found in $P$. vivax infection, affirming that PE biology in the rodent is not an ideal model for human malaria infection. Human clinical trials have identified robust induction of both humoral and cellular immune responses after whole sporozoite immunization yet unequivocal identification of correlates of protection from these studies has proven to be challenging. To reconcile divergent observations, functional in vitro assays have been developed such as the examination of immune sera and its inhibitory activity on infection of cultured hepatomacellines with sporozoites (47-49). Yet, invitrocultured cells do not accurately model the complex architecture of the liver tissue, rendering them only partially physiologically relevant as infection assays. In addition, tissue-resident memory $\mathrm{T}$ cells, which have been shown to be critical in the control of liver stages in rodent malaria infection, do not recirculate into the blood stream (40), impeding the examination of their contribution to PE immunity after $P$. falciparum immunization of humans. Peripheral $\mathrm{T}$ cell populations that correlate with protection have been identified in rodent Plasmodium models (50). However, no ex vivo assays exist to robustly quantify CD8 T cell killing of hepatocytes or the augmentation of humoral and cellular immune responses by CD4 $\mathrm{T}$ cells. Thus, many questions remain regarding the relevance of these cell populations for protection in humans.

\section{HUMANIZED MICE TO MODEL PE IMMUNITY}

Given the lack of robust ex vivo and in vitro assays to functionally examine human immune responses to Plasmodium infection and immunization with candidate vaccines, the identification of true correlates of protection will require the development of animal models that better mimic human Plasmodium infection. Traditionally, this role has been occupied by non-human primates (NHP) where Plasmodium infection better mirrors observations made in humans. Numerous NHP species support infection by Plasmodium species that occur in simians and some NHP support direct infection with $P$. falciparum and $P$. vivax (51). Moreover, the immune response in NHP is similar to humans and tissue-resident immune populations can be sampled to query their importance to vaccine-engendered protection (52). However, NHP systems are still not a perfect model for human Plasmodium infection, and in addition to ethical and financial barriers, logistical concerns regarding housing requirements for NHPs have curtailed their use in malaria research. Thus, the development of immunodeficient mice that have been engrafted with functional human immune cells and human tissues will enable the examination of infection in human cells and tissues in the context of human immune responses in vivo within a small animal model.

Human liver-chimeric mice can play important roles to study functionality of human immune responses. They might serve as an important and cheaper alternative to NHP models in the final down-selection of antibody-based vaccine antigens. For example, we and others have utilized these mice to demonstrate that the passive transfer of human monoclonal antibodies or polyclonal sera from humans immunized with whole $P$. falciparum sporozoites, blocks liver infection (53-55). Of note, mosquito bite challenge of FRG huHep mice after passive transfer allowed superior discrimination of antibody-mediated protection as compared to established in vitro assays. However, given the absence of an adaptive immune response in these mice, active immunization experiments cannot be carried out.

To analyze intrinsic human immune responses, human immune system (HIS) mice have been developed that enable the direct analysis of the HIS responses in vivo after infection with human pathogens or after vaccination. HIS mice are generated by the transplantation of human $\mathrm{CD} 34^{+}$cells containing HSCs into immunodeficient NOG or NSG mice, to model relatively normal human immune responses (56). Huang and colleagues have recently pioneered the generation of HIS mice that possess functional human $\mathrm{CD} 4$, human $\mathrm{CD} 8$, and human $\mathrm{B}$ cell responses $(57,58)$ for the study of PE immunity. To generate humanized mice with a competent human humoral response (referred to as HIS-CD4/B mice), immunocompromised NSG mice were transduced with recombinant adeno-associated virus (AAV) vectors encoding human HLA class II (HLA DR1 or HLA DR4) and a cocktail of human cytokines followed by engraftment with human $\mathrm{CD} 4^{+}$cells (58). To examine the ability of these HIS-CD4/B mice to mount a protective humoral response, HIS-CD4/B mice were first immunized with recombinant $P$. falciparum circumsporozoite protein (CSP) and then subsequently challenged with a transgenic $P$. berghei parasite encoding the repeat regions of $P$. falciparum CSP. Immunized HIS-CD4/B mice exhibited high titers of circulating $P$. falciparum CSP antibodies and showed reduced parasite liver burden after challenge as compared with naive controls (58).

Given the importance of CD8 T cells in PE-engendered protection in rodent models of Plasmodium infection, Huang and colleagues also generated a humanized CD8 T cell mice referred to as HIS-CD8 (57) by transducing NSG mice with AAV vectors encoding functional HLA-A ${ }^{*} 0201$ and a cocktail of human cytokines. Immunization of HIS-CD8 mice with AAV vectors bearing $P$. falciparum CSP resulted in an induction of HLArestricted human CD8 T cells. These immunized HIS-CD8 mice greatly reduced parasite burden in the liver after challenge with transgenic $P$. berghei parasites encoding full length $P$. falciparum CSP (59). Importantly, in vivo depletion of human CD8 T cells completely abolished the reduction in liver burden in immunized HIS-CD8 mice (60).

These HIS studies, however, only support challenge with transgenic rodent malaria parasites expressing selected $P$. falciparum proteins. Thus, there is a need to combine humanized liver-chimeric mice with the HIS models to generate dual-chimeric mice (HIS huHep) susceptible to human Plasmodium liver infection and 
capable of driving functional human immune responses. However, such a model will not yet completely replicate human immunity because although liver-chimeric mice can be repopulated with high levels of huHeps, their liver sinusoidal endothelial cells, Kupffer cells, hepatic stellate cells, and cells of the myeloid lineage all remain of mouse origin. In rodent malaria systems, $\mathrm{CD}^{+}$dendritic cells have been shown to be critical to the generation of an effective immune response after whole sporozoite immunization $(61,62)$. In addition, in $P$. berghei-infected mice, IFNAR expression on myeloid cells is critical for the propagation of the innate immune response to whole parasite infection (42). What roles these cells play in the effective PE immune response to human whole sporozoite immunization remains unknown. Finally, given the importance of liver-resident memory CD8 T cells in PE immunity (38-40), it will be important to examine whether HIS-CD8 mice recapitulate the critical roles of tissueresident cells observed in rodent malaria studies.

\section{HUMANIZED MICE TO MODEL MALARIA BS INFECTION}

Although P. falciparum BSs can be cultured in vitro, a small animal model of human BS malaria would offer great advantages, as it would allow the preclinical testing of drugs and vaccine candidates in an in vivo setting against the human pathogen. The liver-chimeric mice described above support liver infection and liver stage-to-BS transition after injection of target huRBCs $(27,63)$. The presence of huRBCs on the day of exo-erythrocytic merozoite egress from the liver leads to a short period of low parasitemia, and these parasites can then be removed and maintained in huRBC culture. However, BS infection cannot be maintained in the mice as huRBCs are rapidly cleared. Fortunately, different immune-modulation protocols combined with daily injections of huRBCs can support high engraftment levels and promote a continuous $P$. falciparum BS infection in NSG mice (64). These mice show sequestration of the parasite in bone marrow and spleen, suggesting it might resemble the behavior of the parasite in humans. One drawback is that in this study the mice were directly infected with BS parasites, as they are not human liver-chimeric. Moreover, as P. falciparum gametocytes take 10-14 days to mature, this is the time span the infected $\mathrm{RBC}$ has to be maintained to allow transmission back to the mosquito. If this is achieved, the model might enable the study of transmission in an in vivo setting and might allow the testing of transmission blocking drugs and vaccines before moving on to clinical trials. Our laboratory has recently developed a robust protocol to engraft and maintain huRBCs in human liver-chimeric mice to better assess the efficacy of transmission blocking small molecules, antibodies and vaccines (65). Another promising application for combined huHep/huRBC mice is the preclinical evaluation of safety of attenuated $P$. falciparum whole sporozoite vaccine candidates, allowing for exquisite sensitivity in detecting potential breakthrough infection into the blood before testing of new attenuated strains in human trials (66). Furthermore, combined huHep/huRBC mice have been successfully used for the recovery of recombinant parasite progeny from P. falciparum genetic crosses. Previously, such genetic crosses had to be carried out in splenectomized chimpanzees, but it was recently reported that recombinant progeny can also be recovered from FRG huHep mice that had been injected with huRBCs at the time point of merozoite egress from the liver (67). The option to perform genetic crosses in a small animal model provides a robust avenue for forward genetics research and a new avenue to determine the underlying traits of $P$. falciparum drug resistance and other phenotypes of clinical importance.

Whereas $P$. falciparum BSs can be easily cultured in vitro, all efforts to establish a long-term in vitro culture for $P$. vivax have so far met with limited success. Therefore, research on this widespread parasite would especially benefit from a small animal infection model. The distinct feature of $P$. vivax BS parasites is the strong preference for $\mathrm{CD} 1^{+}$reticulocytes (68). These are highly immature erythrocytes that are mainly found in the bone marrow. Consequently, high amounts of $P$. vivax ring stage infected cells are also found in the bone marrow (69). To establish a humanized mouse model that will propagate $P$. vivax BS infection, mice will have to be engrafted with these rare cells. Reticulocytes account for only $0.5-2 \%$ of peripheral blood, of which only a very small fraction are $\mathrm{CD} 71^{+}$. Higher numbers of $\mathrm{CD} 1^{+}$cells are found in umbilical cord blood (UCB) and enriched reticulocytes from UCB have been successfully used for in P. vivax in vitro BS invasion assays (70). It remains to be determined whether the amount of target cells in mice engrafted with the enriched reticulocyte fraction from UCB would be sufficient to propagate a $P$. vivax infection. One avenue to achieve high numbers of $\mathrm{CD} 71^{+}$reticulocytes is to differentiate human HSCs into erythroid precursor cells. These cells should closely resemble $P$. vivax target cells and therefore might support a $P$. vivax $\mathrm{BS}$ infection in vivo. Encouragingly, infection of FRG huHep mice with $P$. vivax sporozoites leads to development of liver stages that undergo full schizogony and release exo-erythrocytic merozoites. When provided with reticulocyte target cells at the time points of release, infection of and development of asexual BSs was observed (27). FRG huHep mice also support hypnozoite persistence, giving hope that a combined liver and blood model for $P$. vivax could one day enable the routine study of relapsing infection (27).

The repeated injection of huRBCs, combined with different immunomodulatory protocols, is a cumbersome process, necessitating an experienced researcher and often leading to losses of mice. An elegant alternative to repeated huRBC reconstitution would be a mouse that intrinsically sustains human erythropoiesis after HSC transplantation. Especially regarding the cell tropism of $P$. vivax described above, a model in which human erythropoiesis takes place in the bone marrow would hopefully provide a setting in which $P$. vivax BSs can develop. Unfortunately, in the humanized mouse models published to date, human erythropoiesis is severely impaired (71). The injection of human cytokines important for human erythropoiesis only leads to a small increase in huRBCs in the periphery (72). A mouse in which human $\mathrm{CD} 4^{+}$HSC transplantation leads to robust human erythropoiesis combined with an HIS, and which in addition harbors a human-chimeric liver, would ultimately enable the production of reproducible data on the developmental life cycle of human malaria parasites. Therefore, it is a high priority goal for malaria research to develop a humanized mouse model, which intrinsically 
promotes human erythropoiesis and can then be combined with one of the human liver-chimeric models described above. Such an advanced humanized mouse model would open up completely unexplored avenues of research. For example, despite its obvious importance, there is an enormous lack of knowledge in the area of Plasmodium coinfections with other pathogens. Malaria and HIV/AIDS especially have a wide geographical overlap, particularly in sub-Saharan Africa, and epidemiological studies have shown cross-contribution to each other's pathogenicity (73), potential antimalarial treatment failure in $\mathrm{HIV}^{+}$patients (74) as well as potential drug-drug interactions (75). Very little is known about the underlying molecular mechanisms of these observations, as we lack a model system to investigate them. Currently, the questions of drug-drug interactions or vaccine safety in coinfected individuals can solely be addressed in clinical trials, as carried out previously for the malaria vaccine candidate RTS, S (76). However, the advanced mouse model described above, with a human-chimeric liver, human erythropoiesis and HIS, could potentially fill this gap.

\section{BS MALARIA PATHOLOGY IN HUMANIZED MICE}

The question of whether a humanized mouse model might enable the study of $P$. falciparum malaria-associated pathophysiology remains largely unexplored. The pathology of severe malaria is mainly determined by adhesion interactions between infected erythrocytes and human endothelial cells. These adhesion interactions lead to the sequestration of infected erythrocytes in the microvasculature, which benefit the parasite by avoiding clearance in the spleen. Unfortunately, this sequestration also leads to vascular occlusion and inflammation, which are important contributors to severe malaria pathology. Three forms of adhesive interactions have been described: the cytoadherence of infected erythrocytes to endothelial cells, formation of rosettes with uninfected erythrocytes and platelet-mediated clumping of infected cells (77). These interactions are mediated by the $P$. falciparum erythrocyte membrane protein 1 (PfEMP1) family, variant antigens expressed on the surface of infected RBCs that interact with multiple host receptors, including ICAM1, CD36, E-selectin, and endothelial protein C receptor (EPCR) (78). There are approximately 60 different variants of PfEMP1, which are encoded by genes of the var family but only expressed one at a time. Depending on which var gene is expressed, the parasite modifies the antigenic properties of infected erythrocytes, which allows it to evade the host immune system but also changes the binding specificity for host receptors. Var gene switching is currently under extensive investigation and a small animal model allowing the controlled in vivo evaluation of this phenomenon would be of great benefit to this important field of research.

The most lethal complication of a $P$. falciparum infection is cerebral malaria. It has been speculated that the targeting of different receptors via the expression of different PfEMP1 variants leads to tissue-specific sequestration of the parasites. In the brain microvasculature, the EPCR has been shown to play an important role in the sequestration of parasites (79). To date, we lack any knowledge of whether infected RBCs sequester in the brain of
P. falciparum-infected humanized mice. It is thus highly relevant to investigate whether the described pathologies of human malaria are recapitulated in any of the humanized mouse models described above. If high huRBC reconstitution and high parasitemia can be achieved in humanized mice it is likely that rosetting of infected erythrocytes will take place in a mouse model. Whether PfEMP1 molecules on infected erythrocytes interact with mouse receptors on endothelial cells remains an unanswered question, however. If this is not the case, a mouse model must be developed in which human receptors are expressed on mouse endothelial cells. If this could be achieved it would open up new possibilities for exploring the potential of anti-adhesion drugs or antibodies as novel malaria therapies.

\section{BS MALARIA IMMUNITY IN HUMANIZED MICE}

Animal models of malaria continue to provide important insights into the immune response to Plasmodium BS infection in general. However, the development of novel immunotherapeutic strategies against BS parasites requires a thorough investigation of the human immune response, particularly to $P$. falciparum BS infection. Our understanding of the human immune response to early BS infection, vaccination, and natural infection has been gleaned from longitudinal studies on subjects enrolled in controlled human malaria infection trials (80-82). Together with the rodent Plasmodium studies, two immunological processes critical for the control of BS malaria infection have been identified, namely, the inflammatory response from innate immune sensing of Plasmodium infections and the humoral response. Plasmodium pathogen-associated molecular patterns engage pattern recognition receptors on innate immune cells and trigger an inflammatory response critical for early parasite control $(83,84)$. However, this inflammatory response can also be pathogenic to the host. Indeed, Plasmodium-engendered type I IFN signaling impairs dendritic cell function in Plasmodium chabaudi (85) and $P$. berghei-infected mice (86) and promotes the production of the immunosuppressive cytokine, IL-10 by Tr1 cells in a human CHMI trial (87). Antibodies have long been known to play a central role in BS malaria immunity (88). Yet, over half a century later, our understanding of the parasite proteins that induce protective antibody responses, the mechanisms of Plasmodiumengendered humoral protection and why protective antibodies only develop after years of repeated exposure is finally maturing. Mounting evidence in rodent models and correlative data in humans from endemic regions have established that Plasmodium evades humoral immunity through dysregulation of $\mathrm{CD}^{+} \mathrm{T}$ cell (89) and B cell dysfunction (90-95). Although the mechanisms behind the roles of inflammation on dendritic cell function and B cell dysfunction have been well studied in rodent models of malaria, a mouse model with a humanized immune system will be critical to confirming whether the mechanisms outlined in the rodent malaria models apply to infection with the human $P$. falciparum parasite. However, as described in preceding sections, the existing humanized mouse models will not accurately mimic a complete HIS as they still contain murine antigen presenting cells, or other murine myeloid cells. Mice that retain human 
antigen presentation and human myeloid cell function will need to be developed to measure these effects.

\section{CONCLUSION}

Conventional mouse model infections with rodent malaria parasites have critically contributed to our understanding of malaria parasite biology, pathogenesis, and immunology and have been important in malaria vaccine and drug discovery. However, differences between the genomes of the human-infective and rodent-infective Plasmodium species as well as significant divergence between mouse and human biology might preclude facile application of knowledge gleaned from traditional rodent systems to the design of effective interventions in humans. Humanized mouse models have emerged as a critical link between traditional rodent models and humans. huHep mice will enable better examination of the factors critical for hepatocyte infection and liver stage development and the understanding of liver stagedirected, infection-preventing interventions. HIS mice are poised to greatly accelerate our understanding of the immune response to human Plasmodium parasites and vaccine candidates as the data gleaned from these studies will more closely represent the immune response in humans. However, current iterations of these HIS mice retain mouse myeloid compartments likely influencing antigen presentation and immune cell residency. Next-generation HIS mice for malaria research will likely require humanization of the liver, bone marrow, lymphoid compartments, and human erythrocytes. BLT (bone marrow, liver, and thymus) mice, where human fetal thymus and liver tissues as well as autologous HSCs are engrafted into the same mouse represent the most complete humanized mouse system to date (96). Combining this with

\section{REFERENCES}

1. Matsuoka H, Yoshida S, Hirai M, Ishii A. A rodent malaria, Plasmodium berghei, is experimentally transmitted to mice by merely probing of infective mosquito, Anopheles stephensi. Parasitol Int (2002) 51:17-23. doi:10.1016/ S1383-5769(01)00095-2

2. Sidjanski S, Vanderberg JP. Delayed migration of Plasmodium sporozoites from the mosquito bite site to the blood. Am J Trop Med Hyg (1997) 57:426-9. doi:10.4269/ajtmh.1997.57.426

3. Vanderberg JP, Frevert U. Intravital microscopy demonstrating antibodymediated immobilisation of Plasmodium berghei sporozoites injected into skin by mosquitoes. Int J Parasitol (2004) 34:991-6. doi:10.1016/j.ijpara.2004. 05.005

4. Mendis K, Sina BJ, Marchesini P, Carter R. The neglected burden of Plasmodium vivax malaria. Am J Trop Med Hyg (2001) 64:97-106. doi:10.4269/ ajtmh.2001.64.97

5. Mueller I, Galinski MR, Baird JK, Carlton JM, Kochar DK, Alonso PL, et al. Key gaps in the knowledge of Plasmodium vivax, a neglected human malaria parasite. Lancet Infect Dis (2009) 9:555-6. doi:10.1016/S1473-3099(09) 70177-X

6. Miller LH, Baruch DI, Marsh K, Doumbo OK. The pathogenic basis of malaria. Nature (2002) 415:673-9. doi:10.1038/415673a

7. Kaushansky A, Kappe SH. Selection and refinement: the malaria parasite's infection and exploitation of host hepatocytes. Curr Opin Microbiol (2015) 26:71-8. doi:10.1016/j.mib.2015.05.013

8. Prudencio M, Mota MM, Mendes AM. A toolbox to study liver stage malaria. Trends Parasitol (2011) 27:565-74. doi:10.1016/j.pt.2011.09.004

9. Matuschewski K. Vaccines against malaria-still a long way to go. FEBS J (2017) 284:2560-8. doi:10.1111/febs.14107
huHep mice would generate the triple-humanized mouse sorely needed for the study of human malaria parasite infection and immunology. However, the high costs and technical demands of such a system will likely preclude it from being widely employed. Ultimately, future humanized mouse models for Plasmodium research will utilize the transplantation of CD $34^{+}$HSC cells to develop robust human immune and erythropoietic compartments and hepatocyte transplantation to ensure human liver chimerism. Ideally, this mouse will in addition harbor human receptors on endothelial cells so that the pathobiology of malaria BS infection can also be modeled. As it will take great effort to create such a complete and complex mouse model, a continued iterative cycle of basic parasitological discovery and immunology in rodent malaria models combined with studies in currently available, albeit imperfect huHep, HIS, and huRBC mice will further increase the predictive value of animal models for human clinical intervention.

\section{AUTHOR CONTRIBUTIONS}

NM and CS prepared the original drafts of this manuscript with subsequent revision by SK.

\section{ACKNOWLEDGMENTS}

Work by the authors is supported by the NIH (SK: R01 AI 114699-01), the German Research Foundation (SCHA 2047/1-1), and the Bill and Melinda Gates Foundation. We thank Dr. Ashley Vaughan and Dr. Brandon Sack for helpful discussions and critical reading of this manuscript.

10. Flannery EL, Chatterjee AK, Winzeler EA. Antimalarial drug discoveryapproaches and progress towards new medicines. Nat Rev Microbiol (2013) 11:849-62. doi:10.1038/nrmicro3138

11. Kaushansky A, Mikolajczak SA, Vignali M, Kappe SH. Of mice in men: the success and promise of humanized mouse models for human malaria parasite infections. Cell Microbiol (2014) 16:602-11. doi:10.1111/cmi.12277

12. Siu E, Ploss A. Modeling malaria in humanized mice: opportunities and challenges. Ann N Y Acad Sci (2015) 1342:29-36. doi:10.1111/nyas.12618

13. Butler NS, Vaughan AM, Harty JT, Kappe SH. Whole parasite vaccination approaches for prevention of malaria infection. Trends Immunol (2012) 33:247-54. doi:10.1016/j.it.2012.02.001

14. Menard R, Tavares J, Cockburn I, Markus M, Zavala F, Amino R. Looking under the skin: the first steps in malarial infection and immunity. Nat Rev Microbiol (2013) 11:701-12. doi:10.1038/nrmicro3111

15. Mota MM, Rodriguez A. Migration through host cells by apicomplexan parasites. Microbes Infect (2001) 3:1123-8. doi:10.1016/S1286-4579(01) 01473-3

16. Risco-Castillo V, Topcu S, Marinach C, Manzoni G, Bigorgne AE, Briquet S, et al. Malaria sporozoites traverse host cells within transient vacuoles. Cell Host Microbe (2015) 18:593-603. doi:10.1016/j.chom.2015.10.006

17. Gueirard P, Tavares J, Thiberge S, Bernex F, Ishino T, Milon G, et al. Development of the malaria parasite in the skin of the mammalian host. Proc Natl Acad Sci U S A (2010) 107:18640-5. doi:10.1073/pnas.1009346107

18. Voza T, Miller JL, Kappe SH, Sinnis P. Extrahepatic exoerythrocytic forms of rodent malaria parasites at the site of inoculation: clearance after immunization, susceptibility to primaquine, and contribution to blood-stage infection. Infect Immun (2012) 80:2158-64. doi:10.1128/IAI.00246-12

19. Guerrero-Aspizua S, Garcia M, Murillas R, Retamosa L, Illera N, Duarte B, et al. Development of a bioengineered skin-humanized mouse model for 
psoriasis: dissecting epidermal-lymphocyte interacting pathways. Am J Pathol (2010) 177:3112-24. doi:10.2353/ajpath.2010.100078

20. Kaushansky A, Douglass AN, Arang N, Vigdorovich V, Dambrauskas N, Kain HS, et al. Malaria parasites target the hepatocyte receptor EphA2 for successful host infection. Science (2015) 350:1089-92. doi:10.1126/science. $\operatorname{aad} 3318$

21. Foquet L, Meuleman P, Hermsen CC, Sauerwein R, Leroux-Roels G. Assessment of parasite liver-stage burden in human-liver chimeric mice. Methods Mol Biol (2015) 1325:59-68. doi:10.1007/978-1-4939-2815-6_5

22. Sacci JB Jr, Alam U, Douglas D, Lewis J, Tyrrell DL, Azad AF, et al. Plasmodium falciparum infection and exoerythrocytic development in mice with chimeric human livers. Int J Parasitol (2006) 36:353-60. doi:10.1016/j. ijpara.2005.10.014

23. Morosan S, Hez-Deroubaix S, Lunel F, Renia L, Giannini C, Van Rooijen N, et al. Liver-stage development of Plasmodium falciparum, in a humanized mouse model. J Infect Dis (2006) 193:996-1004. doi:10.1086/500840

24. Wilson EM, Bial J, Tarlow B, Bial G, Jensen B, Greiner DL, et al. Extensive double humanization of both liver and hematopoiesis in FRGN mice. Stem Cell Res (2014) 13:404-12. doi:10.1016/j.scr.2014.08.006

25. Bissig KD, Wieland SF, Tran P, Isogawa M, Le TT, Chisari FV, et al. Human liver chimeric mice provide a model for hepatitis $\mathrm{B}$ and $\mathrm{C}$ virus infection and treatment. J Clin Invest (2010) 120:924-30. doi:10.1172/JCI40094

26. Vaughan AM, Mikolajczak SA, Wilson EM, Grompe M, Kaushansky A, Camargo N, et al. Complete Plasmodium falciparum liver-stage development in liver-chimeric mice. J Clin Invest (2012) 122:3618-28. doi:10.1172/ JCI62684

27. Mikolajczak SA, Vaughan AM, Kangwanrangsan N, Roobsoong W, Fishbaugher M, Yimamnuaychok N, et al. Plasmodium vivax liver stage development and hypnozoite persistence in human liver-chimeric mice. Cell Host Microbe (2015) 17:526-35. doi:10.1016/j.chom.2015.02.011

28. Soulard V, Bosson-Vanga H, Lorthiois A, Roucher C, Franetich JF, Zanghi G, et al. Plasmodium falciparum full life cycle and Plasmodium ovale liver stages in humanized mice. Nat Commun (2015) 6:7690. doi:10.1038/ncomms8690

29. Nussenzweig RS, Vanderberg J, Most H, Orton C. Protective immunity produced by the injection of $\mathrm{x}$-irradiated sporozoites of Plasmodium berghei. Nature (1967) 216:160-2. doi:10.1038/216160a0

30. Mueller AK, Camargo N, Kaiser K, Andorfer C, Frevert U, Matuschewski K, et al. Plasmodium liver stage developmental arrest by depletion of a protein at the parasite-host interface. Proc Natl Acad Sci U S A (2005) 102:3022-7. doi:10.1073/pnas.0408442102

31. Doll KL, Butler NS, Harty JT. CD8 T cell independent immunity after single dose infection-treatment-vaccination (ITV) against Plasmodium yoelii. Vaccine (2014) 32:483-91. doi:10.1016/j.vaccine.2013.11.058

32. Beaudoin RL. Should cultivated exoerythrocytic parasites be considered as a source of antigen for a malaria vaccine? Bull World Health Organ (1977) 55:373-6.

33. Steel RW, Kappe SH, Sack BK. An expanding toolkit for preclinical preerythrocytic malaria vaccine development: bridging traditional mouse malaria models and human trials. Future Microbiol (2016) 11:1563-79. doi:10.2217/ fmb-2016-0077

34. Potocnjak P, Yoshida N, Nussenzweig RS, Nussenzweig V. Monovalent fragments (Fab) of monoclonal antibodies to a sporozoite surface antigen (Pb44) protect mice against malarial infection. J Exp Med (1980) 151:1504-13. doi:10.1084/jem.151.6.1504

35. Charoenvit Y, Mellouk S, Cole C, Bechara R, Leef MF, Sedegah M, et al. Monoclonal, but not polyclonal, antibodies protect against Plasmodium yoelii sporozoites. J Immunol (1991) 146:1020-5.

36. Sack BK, Kappe SH. A novel immune regulator links malaria and inflammatory bowel disease. J Exp Med (2014) 211. doi:10.1084/jem.21113insight1

37. Keitany GJ, Sack B, Smithers H, Chen L, Jang IK, Sebastian L, et al. Immunization of mice with live-attenuated late liver stage-arresting Plasmodium yoelii parasites generates protective antibody responses to preerythrocytic stages of malaria. Infect Immun (2014) 82:5143-53. doi:10.1128/IAI. 02320-14

38. Tse SW, Radtke AJ, Espinosa DA, Cockburn IA, Zavala F. The chemokine receptor CXCR6 is required for the maintenance of liver memory CD8(+) T cells specific for infectious pathogens. JInfect Dis (2014) 210:1508-16. doi:10.1093/infdis/jiu281
39. Tse SW, Cockburn IA, Zhang H, Scott AL, Zavala F. Unique transcriptional profile of liver-resident memory CD8+ T cells induced by immunization with malaria sporozoites. Genes Immun (2013) 14:302-9. doi:10.1038/gene. 2013.20

40. Fernandez-Ruiz D, Ng WY, Holz LE, Ma JZ, Zaid A, Wong YC, et al. Liver-resident memory $\mathrm{CD} 8(+) \mathrm{T}$ cells form a front-line defense against malaria liver-stage infection. Immunity (2016) 45:889-902. doi:10.1016/j. immuni.2016.08.011

41. McNamara HA, Cai Y, Wagle MV, Sontani Y, Roots CM, Miosge LA, et al. Up-regulation of LFA-1 allows liver-resident memory T cells to patrol and remain in the hepatic sinusoids. Sci Immunol (2017) 2. doi:10.1126/sciimmunol.aaj1996

42. Liehl P, Zuzarte-Luis V, Chan J, Zillinger T, Baptista F, Carapau D, et al. Host-cell sensors for Plasmodium activate innate immunity against liver-stage infection. Nat Med (2014) 20:47-53. doi:10.1038/nm.3424

43. Liehl P, Meireles P, Albuquerque IS, Pinkevych M, Baptista F, Mota MM, et al. Innate immunity induced by Plasmodium liver infection inhibits malaria reinfections. Infect Immun (2015) 83:1172-80. doi:10.1128/IAI.02796-14

44. Miller JL, Sack BK, Baldwin M, Vaughan AM, Kappe SH. Interferonmediated innate immune responses against malaria parasite liver stages. Cell Rep (2014) 7:436-47. doi:10.1016/j.celrep.2014.03.018

45. Zheng H, Tan Z, Zhou T, Zhu F, Ding Y, Liu T, et al. The TLR2 is activated by sporozoites and suppresses intrahepatic rodent malaria parasite development. Sci Rep (2015) 5:18239. doi:10.1038/srep18239

46. Carlton JM, Angiuoli SV, Suh BB, Kooij TW, Pertea M, Silva JC, et al. Genome sequence and comparative analysis of the model rodent malaria parasite Plasmodium yoelii yoelii. Nature (2002) 419:512-9. doi:10.1038/ nature01099

47. Kaushansky A, Rezakhani N, Mann H, Kappe SH. Development of a quantitative flow cytometry-based assay to assess infection by Plasmodium falciparum sporozoites. Mol Biochem Parasitol (2012) 183:100-3. doi:10.1016/j. molbiopara.2012.01.006

48. Finney OC, Keitany GJ, Smithers H, Kaushansky A, Kappe S, Wang R. Immunization with genetically attenuated $P$. falciparum parasites induces long-lived antibodies that efficiently block hepatocyte invasion by sporozoites. Vaccine (2014) 32:2135-8. doi:10.1016/j.vaccine.2014.02.055

49. Seder RA, Chang LJ, Enama ME, Zephir KL, Sarwar UN, Gordon IJ, et al. Protection against malaria by intravenous immunization with a nonreplicating sporozoite vaccine. Science (2013) 341:1359-65. doi:10.1126/science. 1241800

50. Van Braeckel-Budimir N, Harty JT. CD8 T-cell-mediated protection against liver-stage malaria: lessons from a mouse model. Front Microbiol (2014) 5:272. doi:10.3389/fmicb.2014.00272

51. Beignon AS, Le Grand R, Chapon C. In vivo imaging in NHP models of malaria: challenges, progress and outlooks. Parasitol Int (2014) 63:206-15. doi:10.1016/j.parint.2013.09.001

52. Joyner C, Barnwell JW, Galinski MR. No more monkeying around: primate malaria model systems are key to understanding Plasmodium vivax liverstage biology, hypnozoites, and relapses. Front Microbiol (2015) 6:145 doi:10.3389/fmicb.2015.00145

53. Foquet L, Hermsen CC, van Gemert GJ, Van Braeckel E, Weening KE, Sauerwein R, et al. Vaccine-induced monoclonal antibodies targeting circumsporozoite protein prevent Plasmodium falciparum infection. JClin Invest (2014) 124:140-4. doi:10.1172/JCI70349

54. Behet MC, Foquet L, van Gemert GJ, Bijker EM, Meuleman P, Leroux-Roels G, et al. Sporozoite immunization of human volunteers under chemoprophylaxis induces functional antibodies against pre-erythrocytic stages of Plasmodium falciparum. Malar J (2014) 13:136. doi:10.1186/1475-2875-13-136

55. Sack BK, Miller JL, Vaughan AM, Douglass A, Kaushansky A, Mikolajczak S, et al. Model for in vivo assessment of humoral protection against malaria sporozoite challenge by passive transfer of monoclonal antibodies and immune serum. Infect Immun (2014) 82:808-17. doi:10.1128/IAI. 01249-13

56. Ishikawa F. Modeling normal and malignant human hematopoiesis in vivo through newborn NSG xenotransplantation. Int J Hematol (2013) 98:634-40. doi:10.1007/s12185-013-1467-9

57. Huang J, Li X, Coelho-dos-Reis JG, Wilson JM, Tsuji M. An AAV vectormediated gene delivery approach facilitates reconstitution of functional 
human CD8+ T cells in mice. PLoS One (2014) 9:e88205. doi:10.1371/journal. pone. 0088205

58. Huang J, Li X, Coelho-dos-Reis JG, Zhang M, Mitchell R, Nogueira RT, et al. Human immune system mice immunized with Plasmodium falciparum circumsporozoite protein induce protective human humoral immunity against malaria. J Immunol Methods (2015) 427:42-50. doi:10.1016/j.jim.2015.09.005

59. Li X, Huang J, Zhang M, Funakoshi R, Sheetij D, Spaccapelo R, et al. Human CD8+ T cells mediate protective immunity induced by a human malaria vaccine in human immune system mice. Vaccine (2016) 34:4501-6. doi:10.1016/j. vaccine.2016.08.006

60. Li X, Huang J, Kaneko I, Zhang M, Iwanaga S, Yuda M, et al. A potent adjuvant effect of a CD1d-binding NKT cell ligand in human immune system mice. Expert Rev Vaccines (2017) 16:73-80. doi:10.1080/14760584. 2017.1256208

61. Shortman K, Heath WR. The CD8+ dendritic cell subset. Immunol Rev (2010) 234:18-31. doi:10.1111/j.0105-2896.2009.00870.x

62. Cockburn IA, Zavala F. Dendritic cell function and antigen presentation in malaria. Curr Opin Immunol (2016) 40:1-6. doi:10.1016/j.coi.2016.01.010

63. Vaughan AM, Kappe SH, Ploss A, Mikolajczak SA. Development of humanized mouse models to study human malaria parasite infection. Future Microbiol (2012) 7:657-65. doi:10.2217/fmb.12.27

64. Duffier Y, Lorthiois A, Cistero P, Dupuy F, Jouvion G, Fiette L, et al. A humanized mouse model for sequestration of Plasmodium falciparum sexual stages and in vivo evaluation of gametocytidal drugs. Sci Rep (2016) 6:35025. doi:10.1038/srep35025

65. Foquet L, Schafer C, Minkah NK, Alanine DGW, Flannery El, Steel RWJ, et al. Plasmodium falciparum liver stage infection and transition to stable blood stage infection in liver-humanized and blood-humanized FRGN KO mice enables testing of blood stage inhibitory antibodies (reticulocytebinding protein homolog 5) in vivo. Front in Immunol (2018) 9:524. doi:10.3389/fimmu.2018.00524

66. Mikolajczak SA, Lakshmanan V, Fishbaugher M, Camargo N, Harupa A, Kaushansky A, et al. A next-generation genetically attenuated Plasmodium falciparum parasite created by triple gene deletion. Mol Ther (2014) 22: 1707-15. doi:10.1038/mt.2014.85

67. Vaughan AM, Pinapati RS, Cheeseman IH, Camargo N, Fishbaugher M, Checkley LA, et al. Plasmodium falciparum genetic crosses in a humanized mouse model. Nat Methods (2015) 12:631-3. doi:10.1038/nmeth.3432

68. Malleret B, Renia L, Russell B. The unhealthy attraction of Plasmodium vivax to reticulocytes expressing transferrin receptor 1 (CD71). Int J Parasitol (2017) 47:379-83. doi:10.1016/j.ijpara.2017.03.001

69. Baro B, Deroost K, Raiol T, Brito M, Almeida AC, de Menezes-Neto A, et al. Plasmodium vivax gametocytes in the bone marrow of an acute malaria patient and changes in the erythroid miRNA profile. PLoS Negl Trop Dis (2017) 11:e0005365. doi:10.1371/journal.pntd.0005365

70. Russell B, Suwanarusk R, Borlon C, Costa FT, Chu CS, Rijken MJ, et al. A reliable ex vivo invasion assay of human reticulocytes by Plasmodium vivax. Blood (2011) 118:e74-81. doi:10.1182/blood-2011-04-348748

71. Rongvaux A, Willinger T, Martinek J, Strowig T, Gearty SV, Teichmann LL, et al. Development and function of human innate immune cells in a humanized mouse model. Nat Biotechnol (2014) 32:364-72. doi:10.1038/nbt.2858

72. Amaladoss A, Chen Q, Liu M, Dummler SK, Dao M, Suresh S, et al. De novo generated human red blood cells in humanized mice support Plasmodium falciparum infection. PLoS One (2015) 10:e129825. doi:10.1371/journal. pone. 0129825

73. Rattanapunya S, Kuesap J, Chaijaroenkul W, Rueangweerayut R, NaBangchang K. Prevalence of malaria and HIV coinfection and influence of HIV infection on malaria disease severity in population residing in malaria endemic area along the Thai-Myanmar border. Acta Trop (2015) 145:55-60. doi:10.1016/j.actatropica.2015.02.001

74. Bukirwa H, Yeka A, Kamya MR, Talisuna A, Banek K, Bakyaita N, et al. Artemisinin combination therapies for treatment of uncomplicated malaria in Uganda. PLoS Clin Trials (2006) 1:e7. doi:10.1371/journal.pctr. 0010007

75. Kredo T, Bernhardsson S, Machingaidze S, Young T, Louw Q, Ochodo E, et al. Guide to clinical practice guidelines: the current state of play. Int J Qual Health Care (2016) 28:122-8. doi:10.1093/intqhc/mzv115

76. Otieno L, Oneko M, Otieno W, Abuodha J, Owino E, Odero C, et al. Safety and immunogenicity of RTS,S/AS01 malaria vaccine in infants and children with WHO stage 1 or 2 HIV disease: a randomised, double-blind, controlled trial. Lancet Infect Dis (2016) 16:1134-44. doi:10.1016/S14733099(16)30161-X

77. Adams Y, Kuhnrae P, Higgins MK, Ghumra A, Rowe JA. Rosetting Plasmodium falciparum-infected erythrocytes bind to human brain microvascular endothelial cells in vitro, demonstrating a dual adhesion phenotype mediated by distinct $P$. falciparum erythrocyte membrane protein 1 domains. Infect Immun (2014) 82:949-59. doi:10.1128/IAI.01233-13

78. Rowe JA, Claessens A, Corrigan RA, Arman M. Adhesion of Plasmodium falciparum-infected erythrocytes to human cells: molecular mechanisms and therapeutic implications. Expert Rev Mol Med (2009) 11:e16. doi:10.1017/ S1462399409001082

79. Bernabeu M, Smith JD. EPCR and malaria severity: the center of a perfect storm. Trends Parasitol (2017) 33:295-308. doi:10.1016/j.pt.2016. 11.004

80. Sauerwein RW, Roestenberg M, Moorthy VS. Experimental human challenge infections can accelerate clinical malaria vaccine development. Nat Rev Immunol (2011) 11:57-64. doi:10.1038/nri2902

81. Engwerda CR, Minigo G, Amante FH, McCarthy JS. Experimentally induced blood stage malaria infection as a tool for clinical research. Trends Parasitol (2012) 28:515-21. doi:10.1016/j.pt.2012.09.001

82. Tran TM, Samal B, Kirkness E, Crompton PD. Systems immunology of human malaria. Trends Parasitol (2012) 28:248-57. doi:10.1016/j.pt.2012. 03.006

83. Stevenson MM, Riley EM. Innate immunity to malaria. Nat Rev Immunol (2004) 4:169-80. doi:10.1038/nri1311

84. Gazzinelli RT, Kalantari P, Fitzgerald KA, Golenbock DT. Innate sensing of malaria parasites. Nat Rev Immunol (2014) 14:744-57. doi:10.1038/ nri3742

85. Tamura T, Kimura K, Yui K, Yoshida S. Reduction of conventional dendritic cells during Plasmodium infection is dependent on activation induced cell death by type I and II interferons. Exp Parasitol (2015) 159:127-35. doi:10.1016/j.exppara.2015.09.010

86. Haque A, Best SE, de Oca M, James KR, Ammerdorffer A, Edwards CL, et al. Type I IFN signaling in CD8- DCs impairs Th1-dependent malaria immunity. J Clin Invest (2014) 124:2483-96. doi:10.1172/JCI70698

87. Montes, de Oca M, Kumar R, Rivera FL, Amante FH, Sheel M, et al. Type I interferons regulate immune responses in humans with blood-stage Plasmodium falciparum infection. Cell Rep (2016) 17:399-412. doi:10.1016/j. celrep.2016.09.015

88. Cohen S, Butcher GA, Mitchell GH. Mechanisms of immunity to malaria. Bull World Health Organ (1974) 50:251-7.

89. Butler NS, Moebius J, Pewe LL, Traore B, Doumbo OK, Tygrett LT, et al. Therapeutic blockade of PD-L1 and LAG-3 rapidly clears established bloodstage Plasmodium infection. Nat Immunol (2011) 13:188-95. doi:10.1038/ ni. 2180

90. Ryg-Cornejo V, Ioannidis LJ, Ly A, Chiu CY, Tellier J, Hill DL, et al. Severe malaria infections impair germinal center responses by inhibiting $\mathrm{T}$ follicular helper cell differentiation. Cell Rep (2016) 14:68-81. doi:10.1016/j. celrep.2015.12.006

91. Kurup SP, Obeng-Adjei N, Anthony SM, Traore B, Doumbo OK, Butler NS, et al. Regulatory $\mathrm{T}$ cells impede acute and long-term immunity to bloodstage malaria through CTLA-4. Nat Med (2017) 23:1220-5. doi:10.1038/nm. 4395

92. Hansen DS, Obeng-Adjei N, Ly A, Ioannidis LJ, Crompton PD. Emerging concepts in T follicular helper cell responses to malaria. Int J Parasitol (2017) 47:105-10. doi:10.1016/j.ijpara.2016.09.004

93. Krishnamurty AT, Thouvenel CD, Portugal S, Keitany GJ, Kim KS, Holder A, et al. Somatically hypermutated Plasmodium-specific $\operatorname{IgM}(+)$ memory B cells are rapid, plastic, early responders upon malaria rechallenge. Immunity (2016) 45:402-14. doi:10.1016/j.immuni.2016.06.014

94. Zander RA, Obeng-Adjei N, Guthmiller JJ, Kulu DI, Li J, Ongoiba A, et al. PD-1 Co-inhibitory and OX40 Co-stimulatory crosstalk regulates helper $\mathrm{T}$ cell differentiation and anti-Plasmodium humoral immunity. Cell Host Microbe (2015) 17:628-41. doi:10.1016/j.chom.2015.03.007

95. Obeng-Adjei N, Portugal S, Holla P, Li S, Sohn H, Ambegaonkar A, et al. Malaria-induced interferon-gamma drives the expansion of Tbethi atypical memory B cells. PLoS Pathog (2017) 13:e1006576. doi:10.1371/journal. ppat.1006576 
96. Wege AK, Melkus MW, Denton PW, Estes JD, Garcia JV. Functional and phenotypic characterization of the humanized BLT mouse model. Curr Top Microbiol Immunol (2008) 324:149-65.

Conflict of Interest Statement: The authors declare that the research was conducted in the absence of any commercial or financial relationships that could be construed as a potential conflict of interest.
Copyright (c) 2018 Minkah, Schafer and Kappe. This is an open-access article distributed under the terms of the Creative Commons Attribution License (CC BY). The use, distribution or reproduction in other forums is permitted, provided the original author(s) and the copyright owner are credited and that the original publication in this journal is cited, in accordance with accepted academic practice. No use, distribution or reproduction is permitted which does not comply with these terms. 